\title{
Fasciite necrosante por Streptococcus agalactiae em paciente diabética - relato de caso
}

\author{
Necrotizing fasciitis caused by Streptococcus agalactiae in a \\ diabetic patient - case report
}

\author{
Roberta Filipini Rampelotto ${ }^{1,6}$, Thaís Franco Comiz²,6, Rosmari Hörner ${ }^{3,6}$, Rosiéli Martini ${ }^{4,6}$, \\ Cláudia Giacomolli5 ${ }^{5,6}$, Litiérri Razia ${ }^{1,6}$, Melise Nunes ${ }^{1,6}$
}

' Farmacêutica, Mestranda do Programa de Pós-Graduação em Ciências Farmacêuticas da UFSM; ${ }^{2}$ Acadêmica do Curso de Farmácia da UFSM; ${ }^{3}$ Farmacêutica, Mestre em Química, Doutora em Química pela Universidade Federal de Santa Catarina (UFSC), Professora Associada do Departamento de Análises Clínicas e Toxicológicas da UFSM: ${ }^{4}$ Farmacêutica, Mestre e doutoranda pelo PPGFC da UFSM, Técnica Administrativa em Educação na UFSM; ${ }^{5}$ Farmacêutica graduada pelo Centro Universitário Franciscano (UNIFRA), Residente Multiprofissional integrada em Gestão e Atenção Hospitalar no Sistema Público de Saúde, Mestre Profissional em Ciências da Saúde pela UFSM; ${ }^{6}$ Universidade Federal de Santa Maria (UFSM), Santa Maria, RS, Brasil.

\section{RESUMO}

Objetivos: Descrever um caso de fasciite necrosante e choque séptico ocasionado por Streptococcus agalactiae, que acometeu uma paciente com diabetes mellitus. Esta análise foi realizada através dos dados do prontuário e resultados de exames laboratoriais da paciente, que estava internada no Hospital Universitário de Santa Maria, em Santa Maria, Rio Grande do Sul.

Descrição do caso: Paciente do gênero feminino, 73 anos, diagnosticada com diabetes mellitus e cirrose micronodular, foi internada com suspeita de septicemia, simultânea a uma infecção no membro inferior esquerdo, cuja hipótese diagnóstica inicial foi de celulite. Pelas características do quadro clínico, foi feito o diagnóstico de fasciite necrosante. Hemoculturas de dois sítios diferentes positivaram para $S$. agalactiae. A paciente foi a óbito por choque séptico.

Conclusões: $O$ relato deste caso enfatiza a gravidade da fasciite necrosante, que pode ocorrer principalmente em pacientes portadores de fatores predisponentes como diabetes mellitus e cirrose.

DESCRITORES: STREPTOCOCCUS AGALACTIAE; CHOQUE SÉPTICO; FASCIITE NECROSANTE; DIABETES MELLITUS.

\section{ABSTRACT}

Aims: To report an unusual case of necrotizing fasciitis and septic shock caused by Streptococcus agalactiae, which affected a patient with diabetes mellitus. This analysis was performed using data from medical records and laboratory tests results, who was admitted to the University Hospital of Santa Maria, in Santa Maria, Rio Grande do Sul, Brazil.

Case description: Female patient, 73 years old, diagnosed with diabetes mellitus and micronodular cirrhosis, was admitted with suspected septicemia simultaneous with an infection in the left lower limb, which initial diagnostic hypothesis was cellulitis. Based on characteristics of the clinical picture, the diagnosis of necrotizing fasciitis was done. Blood cultures from two different sites were positive for $S$. agalactiae. The patient died with septic shock.

Conclusions: This case report emphasizes the severity of necrotizing fasciitis, which may occur especially in patients with predisposing factors such as diabetes mellitus and cirrhosis.

KEY WORDS: STREPTOCOCCUS AGALACTIAE; SEPTIC SHOCK; NECROTIZING FASCIITIS; DIABETES MELLITUS.

Recebido em janeiro de 2014; aceito em junho de 2014 


\section{INTRODUÇÃO}

A fasciite necrosante (FN) é uma condição clínica aguda grave, de etiologia bacteriana, caracterizada pela necrose progressiva da fáscia superficial do tecido celular subcutâneo com preservação relativa do músculo subjacente. ${ }^{1,2}$ É uma patologia dos tecidos moles, infrequente e de evolução rápida que pode afetar diversas partes do corpo, como genitália, parede torácica, membros, cabeça e pescoço, estando associada a altos índices de morbidade e mortalidade. ${ }^{3-5}$

A FN pode ser classificada em dois tipos: o tipo I, também conhecido por celulite necrosante, ocasionado por flora mista de anaeróbios e outras bactérias, entre elas os estreptococos beta hemolíticos não pertencentes ao grupo A, como Streptococcus agalactiae (também conhecido por Estreptococo do Grupo B); e o tipo II ou gangrena estreptocócica causada por Streptococcus pyogenes (também conhecido por Estreptococo do Grupo A) isolado ou associado ao Staphylococcus aureus. O tipo II ocorre após ferimentos penetrantes, procedimentos cirúrgicos, queimaduras e traumas. ${ }^{6,7}$

Condições debilitantes do sistema imune, como diabetes mellitus (DM), câncer, cirrose, idade avançada e insuficiência renal são os fatores de risco mais comuns associados com $\mathrm{FN}$ e doenças invasivas por $S$. agalactiae, sendo que destas, o DM se destaca pela complicação nos tecidos moles das extremidades inferiores. A FN acomete adultos e idosos e, em seu estágio inicial, apresenta-se como uma infecção superficial das partes moles, como celulite ou erisipela. $\mathrm{Na}$ continuação, manifesta-se com dor intensa, edema grave e rápida progressão, com ausência de resposta a antibioticoterapia isolada, dificultando também o diagnóstico clínico. ${ }^{3,6,8}$ De acordo com Costa et al., ${ }^{9}$ evidências clínicas características de FN incluem edema inelástico, cianose, palidez e hipoestesia cutâneas, crepitação local devida a liberação de gás tecidual (na FN tipo I), fraqueza muscular, odor fétido dos exsudatos, ausência de linfangite, rápida progressão da infecção e falta de resposta à antibioticoterapia convencional.

S. agalactiae é um habitante normal do trato gastrointestinal, mas nas últimas décadas seu envolvimento como agente etiológico de infecções invasivas graves tem se tornado crescente, principalmente em neonatos. Atualmente, vem sendo reportado como agente etiológico de infecções em pacientes adultos e idosos, particularmente em condições debilitantes, tornando-se responsável por significativa morbidade. A partir de uma porta de entrada para a corrente sanguínea, que pode ser a pele, o trato genitourinário ou o trato respiratório, $S$. agalactiae pode causar septicemia. ${ }^{10-13}$

A sepse por $S$. agalactiae apresenta alta incidência nos hospitais, sendo uma das principais causas de morbimortalidade nas unidades de terapia intensiva. ${ }^{14} \mathrm{Na}$ ocorrência de choque séptico, as alterações hemodinâmicas podem não responder à ressussitação, resultando em óbito. Sendo assim, é importante um alto grau de suspeição, para que se possa instituir precocemente o tratamento empírico com antimicrobianos e o debridamento cirúrgico. ${ }^{14-16}$

Este estudo relata um caso de $\mathrm{FN}$ e choque séptico por $S$. agalactiae, diagnosticado no Hospital Universitário de Santa Maria (HUSM), na cidade de Santa Maria, Rio Grande do Sul. O estudo é parte de um projeto que está sendo desenvolvido no HUSM e recebeu aprovação do Comitê de Ética em Pesquisa da Universidade Federal de Santa Maria pelo parecer consubstanciado número 221.889 .

\section{RELATO DO CASO}

Paciente do gênero feminino, 73 anos, realizava exames de rotina no HUSM desde 1995. Em 1999 já apresentava glicemia de jejum de até $219 \mathrm{mg} / \mathrm{dL}$, sendo caracterizada como portadora de DM. Também foi diagnosticada, no mesmo ano, com cirrose hepática micronodular, caracterizada pela substituição difusa da estrutura hepática regular por nódulos de estrutura anormal, rodeados por fibrose. A paciente também apresentava hemocromatose, que entretanto vinha sem acompanhamento há mais de 10 anos.

Em abril de 2013 a paciente procurou auxílio médico no Pronto Atendimento do HUSM, apresentando lesões bolhosas no dorso do pé e na face interna da perna esquerda, áreas de gangrena, extremidades frias e pálidas e exsudato amarelado em toda a superfície da perna. Apresentava também prostração, dor nas articulações, taquicardia e febre $\left(38,9^{\circ} \mathrm{C}\right)$.

Através da anamnese e do exame físico, o clínico levantou inicialmente a hipótese de celulite no membro inferior esquerdo, com possível septicemia. A paciente foi examinada também pelo cirurgião, que suspeitou de FN pelas características apresentadas, sendo indicada a internação hospitalar para realização de exames e tratamento. Foram solicitados eletrocardiograma, exames bioquímicos, exame qualitativo de urina, urocultura e hemocultura, sendo esta última coletada de dois diferentes sítios. Não foi solicitada cultura das lesões de pele.

Antes do resultado do antibiograma, foi iniciado tratamento endovenoso com oxacilina ( $2 \mathrm{~g}$ a cada 
quatro horas), ceftriaxona ( $1 \mathrm{~g}$ a cada 12 horas) e clindamicina (600 mg a cada seis horas). Noradrenalina e dopamina também foram administradas. No mesmo dia da internação, a paciente apresentou características de choque, com hipotensão arterial, insuficiência renal e taquicardia. O eletrocardiograma demonstrou a frequência cardíaca alta (210 batimentos por minuto) com um episódio de fibrilação atrial, sendo cardiovertida com retorno do ritmo sinusal. Ocorreram picos febris de até $39,3{ }^{\circ} \mathrm{C}$ e o hemograma era compatível com infecção bacteriana. Antes de ser possível a intervenção cirúrgica, o quadro clínico de choque séptico agravou-se, e ocorreram disfunção de múltiplos órgãos. Apesar de todas as medidas de controle instituídas, no dia seguinte a paciente apresentou parada cardiorrespiratória irreversível, sendo registrado o óbito.

As duas hemoculturas foram positivas para $S$. agalactiae. O resultado do ensaio de difusão do disco, realizado nos dois isolados, demonstrou o mesmo perfil de sensibilidade. A bactéria apresentava sensibilidade à penicilina, ampicilina, vancomicina, ceftriaxona, linezolida e levofloxacino e resistência à clindamicina e azitromicina, sendo que a oxacilina não foi testada. $\mathrm{O}$ exame qualitativo de urina e a urocultura não demonstraram alterações.

\section{DISCUSSÃO}

S. agalactiae tem sido relatado como agente etiológico de infecções severas, especialmente em pacientes com condições debilitantes. O DM é uma das doenças mais comumente associadas às infecções por $S$. agalactiae em adultos, mas outras condições, incluindo cirrose e idade acima de 65 anos, também estão associadas ao maior risco de doenças invasivas causadas por $S$. agalactiae. ${ }^{8,17-20}$ Além do DM e da idade avançada, esta paciente apresentava cirrose micronodular, a qual se associa com várias complicações clínicas, especialmente peritonite bacteriana espontânea, que acomete cerca de 30\% dos pacientes cirróticos, sendo responsável por altos índices de morbimortalidade. Essa patologia causa prejuízos ao sistema imune, alteração da flora intestinal e translocação bacteriana. ${ }^{21}$

Adicionalmente, a paciente era portadora de hemocromatose, estado clínico caracterizado pelo excesso de ferro no organismo, que afeta diretamente o sistema imune. O ferro em demasia favorece a replicação viral e as infecções bacterianas, deprime a resposta imunológica, induz à fibrogênese e causa lesão celular por liberação de radicais livres. Acredita-se que a disposição para infecções bacterianas ocorra pela disfunção dos linfócitos natural killer e dos macrófagos. O DM é uma das alterações endócrinas associadas à hemocromatose hereditária, desenvolvendo-se em 30 a $60 \%$ dos portadores dessa doença e piorando o seu prognóstico. ${ }^{22-27}$

S. agalactiae pode contaminar a vagina de forma crônica ou intermitente em cerca de um terço das mulheres, sem que cause nenhum sintoma. Sendo assim, a colonização por $S$. agalactiae nas gestantes é comum, podendo a bactéria infectar o recémnascido no momento do parto e resultar na doença estreptocócica neonatal, que é gravíssima e apresenta alta mortalidade. Por isso já existem diretrizes específicas para a pesquisa de rotina da bactéria na gestação e tratamento da parturiente portadora, visando à prevenção da infecção neonatal. . $^{28,29}$ Já a ocorrência de FN por $S$. agalactiae é rara, mas adquire importância por sua letalidade.

As características clínicas da FN, em seu estágio inicial, são inespecíficas, acarretando muitas vezes um diagnóstico tardio, o que pode ocasionar agravamento no estado do paciente e uma progressiva propagação da gangrena. ${ }^{4} \mathrm{O}$ tratamento bem sucedido inclui diagnóstico precoce, intervenção cirúrgica, antibioticoterapia empírica de amplo espectro e medidas gerais de suporte clínico e nutricional, com controle da hipotensão e a avaliação da função dos diversos sistemas que podem ser comprometidos pelo estado séptico..$^{30,31} \mathrm{O}$ diagnóstico e o tratamento precoces reduzem as taxas de morbimortalidade dos pacientes. ${ }^{5}$ Em função da dificuldade no reconhecimento clínico da doença em sua fase inicial, buscam-se exames complementares que possam apontar o risco de FN. Entre estes, existe o escore LRINEC (Laboratory Risk Indicator for Necrotizing Fasciitis), que utiliza um conjunto de resultados de exames bioquímicos e hematológicos. ${ }^{32}$ Pelo fato deste estudo ser retrospectivo, não obtivemos dados suficientes, no prontuário da paciente, que possibilitassem determinar o escore LRINEC neste caso.

S. agalactiae apresenta sensibilidade elevada aos beta-lactâmicos, como penicilina e ampicilina, os quais constituem a antibioticoterapia de eleição. . $^{13,33,34}$ Tratamento alternativo são as cefalosporinas de $2^{\text {a }}$ geração, os macrolídeos e a clindamicina. Em casos de alergia à penicilina, o tratamento pode ser feito com eritromicina. ${ }^{33}$ Porém, tem sido registrada uma relevante resistência à eritromicina e clindamicina. ${ }^{13,34}$ No caso aqui descrito, foram utilizados oxacilina, ceftriaxona e clindamicina, antes do resultado dos antibiogramas. 
A utilização de marcadores de sepse pode facilitar o início precoce do tratamento antibacteriano. Entre estes pode-se citar a procalcitonina, que tem recomendação como um biomarcador específico nas infecções bacterianas. $\mathrm{Na}$ sepse, a procalcitonina apresenta valores acima de $3 \mathrm{ng} / \mathrm{ml}$, enquanto no choque séptico os valores podem ser superiores a $100 \mathrm{ng} / \mathrm{ml}$, sendo que em indivíduos saudáveis seu níveis são indetectáveis. Além disso, a procalcitonina pode ser utilizada como biomarcador de prognóstico, pois a observação dos valores dosados 24 e 48 horas após o início do tratamento mostra-se muito útil na avaliação do prognóstico do paciente, predizendo o desfecho de pacientes com sepse grave ou choque séptico, permitindo assim intervenções e alterações de conduta durante o tratamento. ${ }^{35,36}$
Levando em consideração os aspectos aqui investigados, relatamos um caso de choque séptico em paciente com quadro clínico característico de FN, com duas hemoculturas positivas para S. agalatiae. A cultura da lesão do MIE não foi realizada, constituindo assim, uma limitação deste estudo de caso. No entanto, conforme as referências bibliográficas citadas, os parâmetros clínicos apresentados pela paciente são claros indícios de FN.

De acordo com nosso conhecimento até o momento, este foi o primeiro caso de choque séptico associado a $S$. agalactiae ocorrido neste nosocômio. Assim, este relato chama atenção para a possibilidade desta etiologia e enfatiza a gravidade da FN, principalmente em pacientes portadores de fatores predisponentes.

\section{AGRADECIMENTOS}

Os autores agradecem a toda a equipe do setor de microbiologia do Hospital Universitário de Santa Maria.

\section{REFERÊNCIAS}

1. Swartz MN. Cellulitis and subcutaneous tissue infectious. In: Mandell GL, Bennett JE, Dolin R. Principles and practice of infectious diseases. New York: Churchill Livingstone; 2005. P. 1172-94.

2. Stevens DL, Bisno AL, Chambers HF, Everett ED, Dellinger P, Goldstein EJC, Gorbach SL, Hirschmann JV,Kaplan ED, Montoya JG, Wade JC. Pratice guidelines for the diagnosis and management of skin and soft-tissue infections. Clin Infec Dis 2005;41: $1373-406$

3. Soares FG, Pessanha ALP, Lemos LVB. Fascíte Necrotizante em paciente diabético. Rev Cient Fac Med Campos. 2013;8(2):28-32.

4. Umeda M, Minamikawa T, Komatsubara H, Shibuya Y, Yokoo S, Komori T. Necrotizing fasciitis caused by dental infection: a retrospective analysis of 9 cases and a review of the literature. Oral Surg Oral Med Oral Pathol Oral Radiol Endod. 2003;95:283-90.

5. Costa IMC, Cabral ALSV, Pontes SS, Amorim JF. Fascí́te necrosante: revisão com enfoque nos aspectos dermatológicos. An Bras Dermatol. 2004;79(2):211-24.

6. Neto NT, Giachetto E, Kamamoto F, Ferreira MC. Infecções graves de partes moles: relato de caso de fasciíte necrotizante de face utilizando curativo a vácuo e revisão de literatura. Rev Bras Cir Plást. 2011;26(2):353-9.

7. Fink S, Chaudhuri TK, Davis HH. Necrotizing fasciitis and malpractice claims. South Med J. 1999;92(8):770-4.

8. Wang YW, Su LH, Hou JN, Yang TH, Lin TY, Chu C, Chiu CH. Group B streptococcal disease in nonpregnant patients: emergence of highly resistant strains of serotype Ib in Taiwan in 2006 to 2008. J Clin Microbiol. 2010;48(7):2571-4.

9. Costa IMC, Cabral ALSV, Pontes SS, Amorim JF. Fasciíte necrosante: revisão com enfoque nos aspectos dermatológicos. An Bras Dermatol. 2004;79(2):211-24.

10. Trabulsi L R, Alterthum F. (Org.). Microbiologia. 5a. ed. São Paulo: Atheneu; 2005.

11. Woods CJ, Levy CS, Chandrasekar PH, Talavera F, Brusch JL, Cunha BA. Streptococcus Group B Infections. [Internet]. Medscape; 2009. [cited 2014 Jan 21] Available from: http://emedicine.medscape.com/article/229091-overview

12. Wong SS, Tsui K, Liu Q, Lin L, Tsai CR, Chen L, Huang CH. Serotypes, surface proteins, and clinical syndromes of invasive Group B streptococcal infections in northern Taiwan, 1998-2009. J Microbiol Immunol Infect. 2011;44(1):8-14.

13. Farley MM. Group B streptococcal disease in nonpregnant adults. Clin Infecct Dis 2001;33:556-61.

14. Boechat AL, Boechat NO. Sepse: Diagnóstico e Tratamento. Rev Bras Clin Med. 2010;8:420-7.

15. Figueiredo LPF, Silva E, Corrêa TD. Avaliação hemodinâmica macro e micro-circulatória no choque séptico. Rev Med (São Paulo). 2008;87(2):84-91.

16. Leão LSNO, Passos XS, Reis C, Valadão LMA, Silva MRR, Pimenta FC. Fenotipagem de bactérias isoladas em hemoculturas de pacientes críticos. Rev Soc Bras Med Trop. 2007;40(5):537-40.

17. Baskin LS, Carol PR, Cattolica EV, McAninch JW. Necrotizing soft tissue infections of the perineum and genitalia: bacteriology, treatment, and risk assessment. Br J Urol. 1990;65:524-9. 
18. Borger IL, D’oliveira REC, Castro ACD, Mondino SSB. Streptococcus agalactiae em gestantes: prevalência de colonização e avaliação da suscetibilidade aos antimicrobianos. Rev Bras Ginecol Obstet. 2005;27(10):575-9.

19. Ulett GC, Webb RI, Ulett KB, Cui X, Benjamin WH, Crowley M, Schembri MA. Group B Streptococcus (GBS) Urinary Tract Infection Involves Binding of GBS to Bladder Uroepithelium and Potent but GBS-Specific Induction of Interleukin 1 $\alpha$. J Infect Dis. 2010;201(6): 866-70.

20. Jackson LA, Farley MM, Harrison LH, Reingold AL, Plikaytis BD, Wenger JD, Schuchat A. Risk factors for group B streptococcal disease in adults. Ann Intern Med. 1995;123:415-20.

21. McCord N, Owen P, Powls A, Lunan B. A complete audit cycle of intrapartum group B streptococcus prophylaxis. Health Bull (Edinb). 2001;59:263-7.

22. Adams PC, Valberg LS. Evolving expression of hereditary hemochromatosis. Sem Liver Dis. 1996;16:47-54.

23. Bhavnani M, Lloyd D, Bhattacharyya A, Marples J, Elton P, Worwood M. Screening for genetic haemochromatosis in blood samples with raised alanine aminotransferase. Gut. 2000;46:707-10.

24. Brissot P, Bels F. Current approaches to the management of hemochromatosis. Hematology Am Soc Hematol Educ Program. $2006 ; 36-41$.

25. Hatunic M, Finucane FM, Brennan AM, Norri S, Pacini G, Nolan JJ. Effect of iron overload on glucose metabolism in patients with hereditary hemochromatosis. Metabolism. 2010;59:380-4.

26. Powell LW, Yapp TR. Hemochromatosis. Clin Liver Dis. 2000;4(8):211-28.

27. Souza AFM, Carvalho-Filho RJ, Chebli JF. Hemocromatose hereditária: relato de caso e revisão da literatura. Arq Gastroenterol. 2001;38(3):194-202.

28. Schrag S, Gorwitz R, Fultz-Butts K, Schuchat A. Prevention of perinatal group B streptococcal disease. Revised guidelines from CDC. MMWR Recomm Rep. 2002;51:1-22.

29. Strauss E, Caly WR. Peritonite bacteriana espontânea. Rev Soc Bras Med Trop. 2003;36(6):711-7.

30. Chelsom I, J Halstensen A, Haga T, Hoiby EA. Necrotizing fasciitis due to group A streptococci in Western Norway: incidence and clinical features. Lancet. 1994; 344:1111-5.

31. Soares TH, Penna JTM, Penna LG, Machado JA, Andrade IF, Almeida RC, Vianna LSB. Diagnóstico e tratamento da Fasciíte necrotizante. Rev Med Minas Gerais. 2008;18(2):136-40.

32. Wong CH, Khin LW, Heng KS, Tan KC, Low CO. The LRINEC (laboratory risk indicator for necrotizing fasciitis) score: a tool for distinguishing necrotizing fasciitis from other soft tissue infections. Crit Care Med. 2004;32:1535-41.

33. Panupong L, Chatrchai W. Group B streptococcal bacteremia in nonpregnant adults ats a community teaching hospital. South Med J. 2001;94:1206-11.

34. Manning SD, Foxman B, Pierson CL, Tallman P, Baker CJ, Pearlman MD. Correlates of antibiotic-resistant group B streptococcus isolated from pregnant women. Obstet Gynecol. 2003;1074-9

35. Van den Berghe G, Woulters P, Weekers F, Verwaest C, Bruyninckx F, Schetz M, Vlasselaers D, Ferdinande P, Lauwers P, Bouillon R. Intensive insulin therapy in critically ill patients. N Engl J Med. 2001;345:1359-67.

36. De Azevedo JRA, Torres OJM, Czeczko NG, Tuon FF, Nassif PAN, De Souza GD. Procalcitonina como biomarcador de prognóstico da sepse grave e choque séptico. Rev Col Bras Cir. 2012;39(6):456-61. 